\title{
Image reconstruction in transcranial photoacoustic computed tomography of the brain
}

Kenji Mitsuhashi, Lihong V. Wang, Mark A. Anastasio

Kenji Mitsuhashi, Lihong V. Wang, Mark A. Anastasio, "Image reconstruction in transcranial photoacoustic computed tomography of the brain," Proc. SPIE 9323, Photons Plus Ultrasound: Imaging and Sensing 2015, 93233B (11 March 2015); doi: 10.1117/12.2081050

SPIE. Event: SPIE BiOS, 2015, San Francisco, California, United States 


\title{
Image reconstruction in transcranial photoacoustic computed tomography of the brain
}

\author{
Kenji Mitsuhashi ${ }^{a}$, Lihong V. Wang ${ }^{a}$, and Mark A. Anastasio ${ }^{*} a$ \\ ${ }^{a}$ Department of Biomedical Engineering, Washington University in St. Louis, St. Louis, MO \\ 63130, USA
}

\begin{abstract}
Photoacoustic computed tomography (PACT) holds great promise for transcranial brain imaging. However, the strong reflection, scattering, attenuation, and mode-conversion of photoacoustic waves in the skull pose serious challenges to establishing the method. The lack of an appropriate model of solid media in conventional PACT imaging models, which are based on the canonical scalar wave equation, causes a significant model mismatch in the presence of the skull and thus results in deteriorated reconstructed images. The goal of this study was to develop an image reconstruction algorithm that accurately models the skull and thereby ameliorates the quality of reconstructed images. The propagation of photoacoustic waves through the skull was modeled by a viscoelastic stress tensor wave equation, which was subsequently discretized by use of a staggered grid fourth-order finite-difference time-domain (FDTD) method. The matched adjoint of the FDTD-based wave propagation operator was derived for implementing a backprojection operator. Systematic computer simulations were conducted to demonstrate the effectiveness of the backprojection operator for reconstructing images in a realistic three-dimensional PACT brain imaging system. The results suggest that the proposed algorithm can successfully reconstruct images from transcranially-measured pressure data and readily be translated to clinical PACT brain imaging applications.
\end{abstract}

Keywords: Photoacoustic computed tomography, Image reconstruction, Brain imaging

\section{INTRODUCTION}

Neuroimaging technologies are playing an increasingly important role in the initial detection and subsequent monitoring of a wide range of brain diseases and injuries. The available imaging modalities, however, possess significant shortcomings, e.g. X-ray computed tomography (CT) does not reliably reveal subtle soft-tissue brain features and exposes patients to the long-term effects of ionizing radiation. To overcome these shortcomings, we propose a transcranial neuroimaging modality based on photoacoustic computed tomography (PACT). PACT can exploit the strong optical absorption contrast of injured or diseased brain tissues based on endogenous hemoglobin at high spatial resolution at depths. Its specific advantages over existing neuroimaging modalities include: (1) Safety: PACT does not involve ionizing radiation, (2) Anatomical and functional imaging capabilities: PACT will provide high contrast that is complementary to that revealed by existing methods, (3) Relatively low cost and ease of transport.

Although PACT holds great promise in transcranial neuroimaging, there remain several challenges regarding image reconstruction method designs. In this study, a numerical method necessary to establish an effective image reconstruction algorithm, or more specifically, the matched discrete adjoint of the wave propagation operator, is derived and implemented. The effectiveness of the matched discrete adjoint for reconstructing images in a realistic three-dimensional (3D) PACT brain imaging system is evaluated by use of a numerical simulation study that utilizes a head phantom.

Further additional information contact:

K.M.: kenji.mitsuhashi@wustl.edu

M.A.A.: anastasio@wustl.edu

Photons Plus Ultrasound: Imaging and Sensing 2015, edited by Alexander A. Oraevsky, Lihong V. Wang

Proc. of SPIE Vol. 9323, 93233B - C 2015 SPIE · CCC code: 1605-7422/15/\$18

doi: $10.1117 / 12.2081050$

Proc. of SPIE Vol. 9323 93233B-1 


\section{BACKGROUND}

Below we review the salient features of transcranial PACT imaging physics and the discrete imaging model, which will be used in the following sections. In transcranial PACT, a pulsed laser source is employed to irradiate the brain. ${ }^{1,2}$ The optical energy is rapidly absorbed, resulting in the production of a propagating stress field $\boldsymbol{\sigma}(\mathbf{r}, t)$ and velocity field $\dot{\mathbf{u}}(\mathbf{r}, t)$, where $\mathbf{r} \in \mathbb{R}^{3}$ and $t \in[0, \infty)$ are spatial and temporal coordinates, respectively. Let us assume that the skull possesses spatially-varying linear isotropic elasto-acoustic properties with viscous acoustic absorption characterized by a single absorption coefficient distribution $\alpha(\mathbf{r})$, and the other materials, i.e. the object-to-be-imaged and surrounding medium, possess homogeneous and lossless fluid-like acoustic properties. Additionally, the optical illumination of the object is assumed to be instantaneous, i.e. the laser pulse width is negligible. Under these assumptions, the photoacoustic fields $\boldsymbol{\sigma}(\mathbf{r}, t)$ and $\dot{\mathbf{u}}(\mathbf{r}, t)$ satisfy the elastic wave equation: ${ }^{3}$

$$
\begin{aligned}
& \frac{\partial \dot{\mathbf{u}}(\mathbf{r}, t)}{\partial t}=-\alpha(\mathbf{r}) \dot{\mathbf{u}}(\mathbf{r}, t)+\frac{1}{\rho(\mathbf{r})} \nabla \cdot \boldsymbol{\sigma}(\mathbf{r}, t), \\
& \frac{\partial \boldsymbol{\sigma}(\mathbf{r}, t)}{\partial t}=\lambda(\mathbf{r})(\operatorname{tr} \dot{\boldsymbol{\epsilon}}(\mathbf{r}, t)) \mathbf{I}+2 \mu(\mathbf{r}) \dot{\boldsymbol{\epsilon}}(\mathbf{r}, t),
\end{aligned}
$$

subject to the initial conditions

$$
\left.\boldsymbol{\sigma}(\mathbf{r}, t)\right|_{t=0}=-\frac{\beta c_{0}^{2}}{C_{p}} A(\mathbf{r}) \mathbf{I},\left.\quad \dot{\mathbf{u}}(\mathbf{r}, t)\right|_{t=0}=0,
$$

where $A(\mathbf{r})$ is a compactly supported and bounded function that represents the absorbed optical energy density, $\rho(\mathbf{r})$ is the density, $\lambda(\mathbf{r})$ and $\mu(\mathbf{r})$ are the Lamé parameters, $\mathbf{I}$ is the identity tensor,

$$
\dot{\boldsymbol{\epsilon}}(\mathbf{r}, t) \triangleq \mathcal{S}\left(\frac{\partial \dot{\mathbf{u}}(\mathbf{r}, t)}{\partial \mathbf{r}}\right)
$$

is the strain rate tensor, where $\mathcal{S}(\cdot)$ denotes the symmetric part of the tensor, and $\beta, c_{0}, C_{p}$ are the thermal expansion coefficient, the speed of sound, and the isobaric specific heat of the object, respectively.

The stress field $\boldsymbol{\sigma}(\mathbf{r}, t)$ is subsequently measured with ultrasonic transducer elements arranged on an arbitrary surface enclosing the skull. Since shear waves are supported only in the skull, and all transducer elements are located outside the skull, the stress field measured at the transducer locations is isotropic and thus equivalent to the pressure field $p(\mathbf{r}, t)$, i.e. $\boldsymbol{\sigma}(\mathbf{r}, t)=-p(\mathbf{r}, t) \mathbf{I}$. In a mathematical sense, the goal of transcranial PACT is to determine $A(\mathbf{r})$ from knowledge of $p(\mathbf{r}, t)$ on some measurement aperture outside the skull.

The elastic wave equations (1) and (2) are discretized by use of a staggered grid. ${ }^{3-5}$ The temporal derivatives on the left-hand side of the equations are approximated as second-order temporal finite-differences (FDs), and the spatial derivatives on the right-hand side as fourth-order spatial FDs. The discretized velocity field is defined at the time points $t=0, \Delta t, 2 \Delta t, \ldots$ while the stress field is defined at the time points $t=\frac{1}{2} \Delta t, \frac{3}{2} \Delta t, \frac{5}{2} \Delta t, \ldots$, where $\Delta t$ denotes the sampling interval. At each time step, the velocity field is updated first, and then the stress field is updated based on knowledge of the velocity field. The reader is referred to Ref. 3 for a thorough review of the classical elastic FDTD method.

The photoacoustic fields are assumed to be defined in the entire $\mathbb{R}^{3}$ space in this study. To simulate these fields using a finite-size computational volume, the unsplit convolutional perfectly-matched layers (CPML) technique $^{6}$ is employed to avoid artificial reflection and/or distortion of photoacoustic waves at the boundary of the computational volume. The technique introduces absorbing boundary layers that each modify the spatial derivatives along the direction perpendicular to the layer as

$$
\partial_{\xi}=\frac{1}{\kappa_{\xi}} \partial_{\xi}+\psi_{\xi}
$$

where the subscript $\xi$ denotes the direction, $\kappa_{\xi} \geq 1$ is a damping coefficient, and $\psi_{\xi}$ is a memory variable that satisfies the time-evolution equation

$$
\psi_{\xi}^{(n+1)}=b_{\xi} \psi_{\xi}^{(n)}+a_{\xi}\left(\partial_{\xi}\right)^{\left(n+\frac{1}{2}\right)},
$$


where the superscript " $(n)$ " denotes the time step, and $a_{\xi}$ and $b_{\xi}$ are defined as

$$
a_{\xi}=\frac{d_{\xi}}{\kappa_{\xi}\left(d_{\xi}+\kappa_{\xi} \alpha_{\xi}\right)}\left(b_{\xi}-1\right), \quad b_{\xi}=\mathrm{e}^{-\left(\frac{d_{\xi}}{\kappa_{\xi}}+\alpha_{\xi}\right) \Delta t} .
$$

Here, $d_{\xi} \geq 0$ and $\alpha_{\xi} \geq 0$ are damping coefficients. We refer the reader to Ref. 6 for the additional details of the CPML technique, e.g. the optimal values of the damping coefficients.

\section{THEORY OF MATCHED DISCRETE ADJOINT}

The adjoint of the imaging operator is most commonly derived from a continuous formulation and subsequently discretized by sampling. However, the adjoint derived in this manner does not form an adjoint pair with the discrete imaging operator and thus may result in deteriorated reconstructed images. ${ }^{7,8}$ In this study, the adjoint is derived from a discrete formulation for the imaging operator that incorporates an inhomogeneous absorptive medium model as follows.
$\dot{u}^{i}(\mathbf{r}, t) \quad$ The particle velocity in the $i$ th direction
$\sigma^{j k}(\mathbf{r}, t) \quad$ The stress in the $j$ th direction acting on a plane perpendicular to the $k$ th direction
$\psi^{i, l}(\mathbf{r}, t) \quad$ The memory variable for the spatial derivative of $\dot{u}^{i}(\mathbf{r}, t)$ in the $l$ th direction
$\phi^{j k}(\mathbf{r}, t) \quad$ The memory variable for the spatial derivative of $\sigma^{j k}(\mathbf{r}, t)$ in the $k$ th direction
$\mathbf{r}_{n}^{i} \quad$ The location of the $n$th spatial grid point at which $\dot{u}^{i}(\mathbf{r}, t)$ is sampled
$\mathbf{r}_{n}^{j k} \quad$ The location of the $n$th spatial grid point at which $\sigma^{j k}(\mathbf{r}, t)$ is sampled
$t_{m} \quad$ The $m$ th temporal grid point, i.e. $t_{m}=m \Delta t$
$\rho(\mathbf{r}) \quad$ The density of the medium
$\lambda(\mathbf{r}), \mu(\mathbf{r}) \quad$ The Lamé parameters of the medium
$\alpha(\mathbf{r}) \quad$ The diffusive absorption coefficient of the medium
$1(\mathbf{r}) \quad$ The unity function defined in the medium
$a^{i}(\mathbf{r}), b^{i}(\mathbf{r}) \quad$ The PML decay constants in the $i$ th direction
$\chi^{l}(\mathbf{r}) \quad$ The PML indicator function for layers perpendicular to the $l$ th direction
$\boldsymbol{\nabla}_{i, l} \quad$ The FD operator in the $l$ th direction acting on a variable sampled at $\left\{\mathbf{r}_{n}^{i}\right\}$
$\nabla_{j k} \quad$ The FD operator in the $k$ th direction acting on a variable sampled at $\left\{\mathbf{r}_{n}^{j k}\right\}$
Table 1. List of primary variables and operators.

Derived variables and operators Important variables and operators derived from the primary variables and operators (Table 1) as follows:

$$
\begin{aligned}
\dot{\mathbf{u}}_{m-\frac{1}{2}}^{i} & =\left(\dot{u}^{i}\left(\mathbf{r}_{1}^{i}, t_{m-\frac{1}{2}}\right), \ldots, \dot{u}^{i}\left(\mathbf{r}_{N}^{i}, t_{m-\frac{1}{2}}\right)\right)^{\mathrm{T}} \\
\boldsymbol{\psi}_{m-\frac{1}{2}}^{i, l} & =\left(\psi^{i, l}\left(\mathbf{r}_{1}^{i l}, t_{m-\frac{1}{2}}\right), \ldots, \psi^{i, l}\left(\mathbf{r}_{N}^{i l}, t_{m-\frac{1}{2}}\right)\right)^{\mathrm{T}} \\
\boldsymbol{\sigma}_{m}^{j k} & =\left(\sigma^{j k}\left(\mathbf{r}_{1}^{j k}, t_{m}\right), \ldots, \sigma^{j k}\left(\mathbf{r}_{N}^{j k}, t_{m}\right)\right)^{\mathrm{T}} \\
\boldsymbol{\phi}_{m}^{j k} & =\left(\phi^{j k}\left(\mathbf{r}_{1}^{j}, t_{m}\right), \ldots, \phi^{j k}\left(\mathbf{r}_{N}^{j}, t_{m}\right)\right)^{\mathrm{T}} \\
\mathbf{A}_{i, l} & =\operatorname{diag}\left(a^{l}\left(\mathbf{r}_{1}^{i l}\right), \ldots, a^{l}\left(\mathbf{r}_{N}^{i l}\right)\right) \\
\mathbf{B}_{i, l} & =\operatorname{diag}\left(b^{l}\left(\mathbf{r}_{1}^{i l}\right), \ldots, b^{l}\left(\mathbf{r}_{N}^{i l}\right)\right) \\
\mathbf{A}_{j k} & =\operatorname{diag}\left(a^{k}\left(\mathbf{r}_{1}^{j}\right), \ldots, a^{k}\left(\mathbf{r}_{N}^{j}\right)\right) \\
\mathbf{B}_{j k} & =\operatorname{diag}\left(b^{k}\left(\mathbf{r}_{1}^{j}\right), \ldots, b^{k}\left(\mathbf{r}_{N}^{j}\right)\right) \\
\mathbf{D}_{i} & =\Delta t \operatorname{diag}\left(\alpha\left(\mathbf{r}_{1}^{i}\right), \ldots, \alpha\left(\mathbf{r}_{N}^{i}\right)\right) \\
\mathbf{I}_{i} & =\operatorname{diag}\left(1\left(\mathbf{r}_{1}^{i}\right), \ldots, 1\left(\mathbf{r}_{N}^{i}\right)\right) \\
\mathbf{I}_{j k} & =\operatorname{diag}\left(1\left(\mathbf{r}_{1}^{j k}\right), \ldots, 1\left(\mathbf{r}_{N}^{j k}\right)\right) \\
\mathbf{J}_{i} & =\mathbf{I}_{i}-\mathbf{D}_{i}
\end{aligned}
$$




$$
\begin{aligned}
\mathbf{Q}_{i} & =\operatorname{diag}\left(\rho\left(\mathbf{r}_{1}^{i}\right), \ldots, \rho\left(\mathbf{r}_{N}^{i}\right)\right) \\
\mathbf{\Lambda}_{j k} & =\operatorname{diag}\left(\lambda\left(\mathbf{r}_{1}^{j k}\right), \ldots, \lambda\left(\mathbf{r}_{N}^{j k}\right)\right) \\
\mathbf{M}_{j k} & =\operatorname{diag}\left(\mu\left(\mathbf{r}_{1}^{j k}\right), \ldots, \mu\left(\mathbf{r}_{N}^{j k}\right)\right) \\
\mathbf{X}_{i, l} & =\operatorname{diag}\left(\chi^{l}\left(\mathbf{r}_{1}^{i l}\right), \ldots, \chi^{l}\left(\mathbf{r}_{N}^{i l}\right)\right) \\
\mathbf{X}_{j k} & =\operatorname{diag}\left(\chi^{k}\left(\mathbf{r}_{1}^{j}\right), \ldots, \chi^{k}\left(\mathbf{r}_{N}^{j}\right)\right) \\
\boldsymbol{\Gamma}_{i ; j k} & =\Delta t \delta_{i j} \mathbf{Q}_{i}^{-1} \mathbf{X}_{j k} \\
\boldsymbol{\Delta}_{j k ; i l} & =\Delta t\left[\delta_{j k} \delta_{i l} \mathbf{\Lambda}_{j k}+\left(\delta_{i j} \delta_{k l}+\delta_{i k} \delta_{j l}\right) \mathbf{M}_{j k}\right] \mathbf{X}_{i, l} \\
\mathbf{\Phi}_{i ; j k} & =\Delta t \delta_{i j} \mathbf{Q}_{i}^{-1} \boldsymbol{\nabla}_{j k} \\
\mathbf{\Psi}_{j k ; i} & =\Delta t\left[\delta_{j k} \boldsymbol{\Lambda}_{j k} \boldsymbol{\nabla}_{i, i}+\mathbf{M}_{j k}\left(\delta_{i j} \boldsymbol{\nabla}_{i, k}+\delta_{i k} \boldsymbol{\nabla}_{i, j}\right)\right] \\
\mathbf{E}_{i, l} & =\mathbf{A}_{i, l} \boldsymbol{\nabla}_{i, l} \\
\mathbf{E}_{j k} & =\mathbf{A}_{j k} \boldsymbol{\nabla}_{j k}
\end{aligned}
$$

Forward scheme Eqs. (31)-(34) are recursively applied to the wavefield variables in order. When implemented as an algorithm that parallelly updates the values of multiple computational volumes (CVs), the scheme will require a data exchange between two adjacent CVs after the application of Eqs. (32) and (34).

$$
\begin{aligned}
\phi_{m}^{j k} & =\mathbf{B}_{j k} \boldsymbol{\phi}_{m-1}^{j k}+\mathbf{E}_{j k} \boldsymbol{\sigma}_{m}^{j k} \\
\dot{\mathbf{u}}_{m+\frac{1}{2}}^{i} & =\mathbf{J}_{i} \dot{\mathbf{u}}_{m-\frac{1}{2}}^{i}+\sum_{1 \leq j, k \leq 3}\left(\boldsymbol{\Phi}_{i ; j k} \boldsymbol{\sigma}_{m}^{j k}+\boldsymbol{\Gamma}_{i ; j k} \boldsymbol{\phi}_{m}^{j k}\right) \\
\boldsymbol{\psi}_{m+\frac{1}{2}}^{i, l} & =\mathbf{B}_{i, l} \boldsymbol{\psi}_{m-\frac{1}{2}}^{i, l}+\mathbf{E}_{i, l} \dot{\mathbf{u}}_{m+\frac{1}{2}}^{i} \\
\boldsymbol{\sigma}_{m+1}^{j k} & =\boldsymbol{\sigma}_{m}^{j k}+\sum_{1 \leq i \leq 3}\left(\boldsymbol{\Psi}_{j k ; i} \dot{\mathbf{u}}_{m+\frac{1}{2}}^{i}+\sum_{1 \leq l \leq 3} \boldsymbol{\Delta}_{j k ; i, l} \boldsymbol{\psi}_{m+\frac{1}{2}}^{i, l}\right)
\end{aligned}
$$

Backward scheme The backward scheme was derived from Eqs. (31)-(34) in a similar manner to that described in Refs. 7 and 8.

\section{COMPUTER-SIMULATION STUDIES}

A numerical head phantom with a skull model based on the CT data of a human skull was employed. The acoustic properties of the skull were determined by following the procedure described in Ref. 9. The simulated pressure data were generated by use of the FDTD-based wave propagation operator. ${ }^{3,5,10}$

The image reconstructed by use of the widely-employed conventional back-projection type image reconstruction algorithm, namely UBP, ${ }^{11}$ and the image reconstructed by use of the FDTD-based image reconstruction algorithm were compared (Fig. 1). The image reconstructed by use of the FDTD-based image reconstruction algorithm possessed fewer artifacts than the image reconstructed by use of the UBP algorithm.

\section{CONCLUSIONS}

PACT holds great promise in transcranial neuroimaging. Its specific advantages over existing neuroimaging modalities include: (1) Safety: PACT does not involve ionizing radiation, (2) Anatomical and functional imaging capabilities: PACT can exploit the strong optical absorption contrast of injured or diseased brain tissues based on endogenous hemoglobin at high spatial resolution at depths, which is complementary to that revealed by existing methods, (3) Relatively low cost and ease of transport. However, there remain challenges to realize this promise.

In this study, a numerical method necessary to establish an effective image reconstruction algorithm, or more specifically, the matched discrete adjoint of the wave propagation operator, was derived and implemented. The 


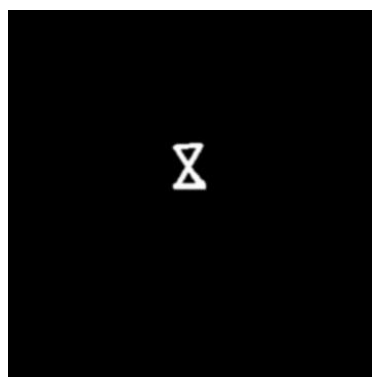

True

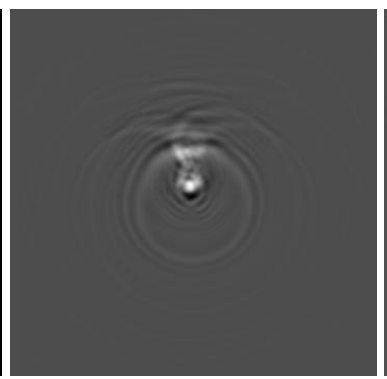

UBP

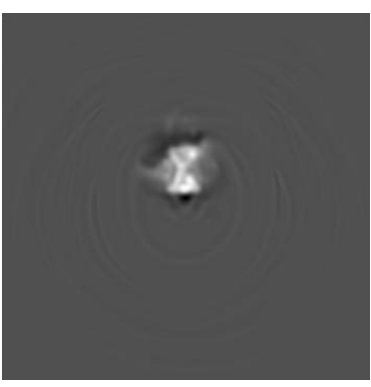

Adjoint

Figure 1. Comparison of the image reconstructed by use of the UBP and the images reconstructed by use of the FDTDbased image reconstruction algorithms. Gray scale: From black (minimum) to white (maximum).

effectiveness of the matched discrete adjoint for reconstructing images in a realistic three-dimensional (3D) PACT brain imaging system was evaluated by use of a numerical simulation study that utilizes a head phantom. The image reconstructed by use of the FDTD-based image reconstruction algorithm possessed fewer artifacts than the image reconstructed by use of the widely-employed conventional back-projection type algorithm. These results suggest that the proposed image reconstruction algorithm can effectively reconstruct images from transcraniallycollected pressure data and readily be translated to actual PACT neuroimaging applications.

\section{ACKNOWLEDGMENTS}

This work was supported in part by NIH awards CA167446 and EB01696301.

\section{REFERENCES}

[1] Wang, L. V., "Multiscale photoacoustic microscopy and computed tomography," Nat. Photonics 3(9), 503509 (2009).

[2] Nie, L., Cai, X., Maslov, K. I., Garcia-Uribe, A., Anastasio, M. A., and Wang, L. V., "Photoacoustic tomography through a whole adult human skull with a photon recycler," J. Biomed. Opt. 17(11), 110506110506 (2012).

[3] Moczo, P., Kristek, J., Galis, M., Pazak, P., and Balazovjech, M., "The finite-difference and finite-element modeling of seismic wave propagation and earthquake motion," Acta Phys. Slovaca 57(2), 177-406 (2007).

[4] Moczo, P., Kristek, J., Vavryčuk, V., Archuleta, R. J., and Halada, L., "3D heterogeneous staggered-grid finite-difference modeling of seismic motion with volume harmonic and arithmetic averaging of elastic moduli and densities," B. Seismol. Soc. Am. 92(8), 3042-3066 (2002).

[5] Michéa, D. and Komatitsch, D., "Accelerating a three-dimensional finite-difference wave propagation code using GPU graphics cards," Geophys. J. Int. 182(1), 389-402 (2010).

[6] Komatitsch, D. and Martin, R., "An unsplit convolutional perfectly matched layer improved at grazing incidence for the seismic wave equation," Geophysics 72(5), SM155-SM167 (2007).

[7] Ji, J., "An exact adjoint operation pair in time extrapolation and its application in least-squares reverse-time migration," Geophysics 74(5), H27-H33 (2009).

[8] Huang, C., Wang, K., Nie, L., Wang, L. V., and Anastasio, M. A., "Full-wave iterative image reconstruction in photoacoustic tomography with acoustically inhomogeneous media," IEEE Trans. Med. Imaging 32(6), 1097-1110 (2013).

[9] Pinton, G., Aubry, J. F., Bossy, E., Muller, M., Pernot, M., and Tanter, M., "Attenuation, scattering, and absorption of ultrasound in the skull bone," Med. Phys. 39(1), 299-307 (2012).

[10] Micikevicius, P., "3D finite difference computation on GPUs using CUDA," in [Proc. GPGPU-2], 79-84, $\mathrm{ACM}(2009)$.

[11] Xu, M. and Wang, L. V., "Universal back-projection algorithm for photoacoustic computed tomography," Phys. Rev. E 71(1), 016706 (2005). 